\title{
PREDICTIVE MODEL FOR SURVIVAL IN PATIENTS WITH GASTRIC CANCER
}

\author{
Amin Golabpoor ${ }^{1}$, Alireza Khooie ${ }^{2}$, Zahra Yousefli ${ }^{3}$, Naieme Farhang Nezhad ${ }^{3}$, Ramin Hosein Zadeh ${ }^{3}$, Sanaz
} Nastarani $^{3}$, Kobra Etminani ${ }^{1}$, Ladan Goshayeshi ${ }^{4, *}$

1: Department of Medical Informatics, Faculty of Medicine, Mashhad University of Medical Sciences, Mashhad, Iran

2: Pathology Department, Mashhad University of Medical Sciences, Mashhad, Iran.

3: Medical Student, Student Research Committee, Faculty of Medicine, Mashhad University of Medical Sciences, Mashhad, Iran

4: Surgical oncology research center, Faculty of Medicine, Mashhad University of Medical Sciences, Mashhad, Iran

Correspondence:

Ladan Goshayeshi. Tel: +989155109704, E-mail: ladangosh@yahoo.com

\section{TYPE OF ARTICLE: CONFERENCE ABSTRACT}

\begin{abstract}
Introduction: Gastric cancer is one of the most prevalent cancers in the world characterized by poor prognosis and is a frequent cause of cancer in Iran. The aim of the study is to conduct a predictive model of survival time for patients suffering from gastric cancer.

Methods: Fifteen factors were analyzed retrospectively in 277 patients by gathering data from the Iranian Cancer Registry in Mashhad, Iran, and also by interviewing with those patients or their relatives. Missing values were imputed by data mining techniques. Survival was taken into account as a dependent variable. Then, the predictive model was conducted by combining both genetic algorithms and logistic regression. MATLAB 2012 software was used to combine them.

Results: Of the 277 patients, only survival of 80 patients was available; their data were used for conducting predictive model. Mean \pm SD of missing values for each patient was $6.02 \pm 2.11$. The combined predictive model achieved $72.57 \%$ accuracy. Sex, birth year, age at diagnosis time, age of diagnosis time at patient's family, family history of gastric cancer, and family history of other gastrointestinal cancers were six parameters associated with patient survival.

Conclusion: The study revealed that imputing missing values by data mining techniques have good accuracy. We also revealed six parameters extracted by genetic algorithms that affect the survival of patients with gastric cancer. Our combined predictive model with a good accuracy is appropriate to forecast survival of patients suffering from gastric cancer. Thus, we suggest policymakers and specialists to use it for predicting patient's survival.

KEYWORDS: Gastric cancer, Survival, Missing value, Predictive model
\end{abstract}

\section{Abstracts of First National Congress of Medical Informatics, Mashhad, Iran, February 2017}

(C) 2017 The Authors. This is an open access article under the terms of the Creative Commons Attribution-NonCommercialNoDerivs License, which permits use and distribution in any medium, provided the original work is properly cited, the use is non-commercial and no modifications or adaptations are made. 